\title{
FREE CARRIER CONCENTRATION DEPENDENCE OF Gd-Gd EXCHANGE CONSTANT IN SnTe:Gd*
}

\author{
A. LUSAKOWSKI \\ Institute of Physics, Polish Academy of Sciences \\ Al. Lotników 32/46, 02-668 Warszawa, Poland
}

\begin{abstract}
In some samples of SnTe:Gd the measured paramagnetic Curie temperature strongly depends on the Fermi level position. The semi-quantitative theory which explains this effect is presented. The theory is based on Kasuya's model of exchange interaction between two $4 f$ spins of rare earth ions. In this model the interaction between $4 f$ electrons and the valence band goes via $\mathrm{Gd}$ atomic $5 d$ orbitals which, contrary to the $4 f$ orbitals are much more extended in space. The exchange integral and the paramagnetic Curie temperature dependence on the Fermi level position are calculated.

PACS numbers: $71.70 . \mathrm{Gm}, 75.30 . \mathrm{Et}, 75.30 . \mathrm{Hx}$
\end{abstract}

\section{Introduction}

The experimental investigations of $\mathrm{SnTe}$ Gd revealed the fact that the magnetic properties of some samples (it depends on Gd content) strongly depend on the free carrier concentration $[1,2]$. In order to understand this behavior, we consider the mechanism of exchange interaction between two $\mathrm{Gd}$ atoms proposed by Kasuya [3], in which the interaction between the $4 f$ electrons and the valence band goes via Gd atomic $5 d$ orbital. However, contrary to Kasuya, we consider hybridization of $5 d$ orbital not only with the $p$ orbital of the intervening anion but with all the valence band. This enables us to take into account the position of the Fermi level (concentration of free carriers) and the distance between two gadolinium atoms.

\section{The Hamiltonian}

Let us consider two gadolinium atoms in SnTe placed at $\boldsymbol{R}_{1}$ and $\boldsymbol{R}_{2}$. In analogy to the Kasuya model [3] the Hamiltonian of the system consists of four terms

$$
H=H_{0}+H_{d}+H_{d j}+H_{p d},
$$

*This work was supported by the State Committee for Scientific Research (Republic of Poland) under grant No. 2 PO3B 10308. 
where

and

$$
H_{0}=\sum_{k, \alpha} \epsilon_{k} a_{k \alpha}^{\dagger} a_{k \alpha}
$$

$$
H_{d}=\sum_{i, \alpha} \epsilon_{d} d_{i \alpha}^{\dagger} d_{i \alpha}
$$

correspond to band and $d$ electrons of Gd atom respectively. We assume that the level we call $d$ level is only doubly degenerate with respect to the spin. The intra-atomic coupling between $d$ electrons and the spins of the $f$ shells is described by

Finally

$$
H_{d f}=I \sum_{i, \alpha, \beta} S_{i} d_{i \alpha}^{\dagger} \sigma_{\alpha \beta} d_{i \beta} .
$$

$$
H_{p d}=\sum_{k_{\alpha i \beta}}\left(V_{k_{\alpha, i \beta}} a_{k_{\alpha}}^{\dagger} d_{i \beta}+V_{k_{\alpha, i \beta}}^{*} d_{i \beta}^{\dagger} a_{k_{\alpha}}\right)
$$

is the hybridization between $d$ levels and the valence band.

\section{Assumptions and approximations}

1. We treat the spin of the gadolinium as a classical quantity, $S=7 / 2$.

2. We consider a situation with the Fermi level position in $\Sigma$ band and we neglect the influence of the other bands. The $\Sigma$ band consists of twelve valleys placed at $K_{0}(1,1,0), K_{0}(-1,1,0)$ and so on. The value of $K_{0}$ is known only approximately [4], in the following $K_{0}=3 \pi / 4 a_{0}$, where $a_{0}=6.33 \AA$ is the lattice constant. The dispersion relation in the vicinity of the extremum at $K_{0}(1,1,0)$, for example, reads

$$
\epsilon_{\boldsymbol{k}}=-\left(\hbar^{2} / 2 m\right)\left[\left(k_{x}-K_{0}\right)^{2}+\left(k_{y}-K_{0}\right)^{2}+A k_{z}^{2}\right],
$$

where $m=0.2 m_{0}$ and the factor $A=0.1$ [4] takes care on the band's anisotropy. The analogous formulas may be written for the remaining $\Sigma$ valleys. We neglect the spin-orbit interaction.

3. The matrix elements between the $d$ level and the band are of the form

$$
V_{\boldsymbol{k} \alpha, i \beta}=1 / \sqrt{N} \exp \left(\mathrm{i} \boldsymbol{k} \cdot \boldsymbol{R}_{i}\right) V \delta_{\alpha \beta}
$$

( $N$ is the number of elementary cells in the crystal), which means that we consider only transitions without change of the electron's spin. This simple form with $k$ independent $V$ is valid if the $d$ level is very well localized in space. In more quantitative theory the $k$ dependence of $V$ should be taken into account. In the present work we treat $V$ as the fitting parameter.

4. We assume that $V \ll I S(I S \approx 1 \mathrm{eV})$.

5. Due to the intra-atomic $H_{d f}$ interaction the degeneracy of the $d$ level is lifted. We obtain two levels, the energies of which are $\epsilon_{d} \pm I S$. (The energy is measured with respect to the top of the $\Sigma$ band.) We assume that the energy of one of them $\epsilon_{d}+I S \equiv-\epsilon_{0}<0$. This is precisely that level which is responsible for the interaction between two gadolinium atoms.

6 . We consider the situation when the Fermi energy $\mu \equiv-\mu_{0}<-\epsilon_{0}$. 


\section{The constrained grand canonical potential $\Omega_{0}\left(S_{1}, S_{2}, \mu, T\right)$}

Because the Hamiltonian $H$ is bilinear in creation and annihilation electron operators it is possible to integrate over electron degrees of freedom in the formula for the grand partition function

$$
Z=\int d S_{1} d S_{2} \operatorname{Tr}_{\mathrm{el}} \exp [-\beta(H-\mu n)] .
$$

The method of calculation of $\operatorname{Tr}_{\mathrm{el}}$ is described in [5]. As a result one obtains the quantity $\Omega_{0}\left(S_{1}, S_{2}, \mu, T\right)$ which is related to the grand partition function by the equation

$$
Z=Z_{\text {el }} \int d S_{1} d S_{2} \exp \left[-\beta \Omega_{0}\left(S_{1}, S_{2}, \mu, T\right)\right],
$$

where $Z_{\mathrm{el}}$ is the grand partition function in the absence of $\mathrm{Gd}$ atoms. $\Omega_{0}\left(S_{1}, S_{2}, \mu, T\right)$ describes the effective spin-spin interaction and contains all the information about the band structure, exchange integral $I$ and matrix elements $V_{\boldsymbol{k} \alpha, i \beta}$. In the first approximation with respect to the product $\boldsymbol{S}_{1} \cdot \boldsymbol{S}_{2}$,

$$
\Omega_{0}\left(\boldsymbol{S}_{1}, \boldsymbol{S}_{2}, \mu, T\right) \approx \operatorname{const}(S, \mu, T)+J(\boldsymbol{R}, \mu, T) \boldsymbol{S}_{1} \cdot \boldsymbol{S}_{2} \quad\left(\boldsymbol{R}=\boldsymbol{R}_{1}-\boldsymbol{R}_{2}\right) .
$$

This approximation is valid if the difference between the Fermi and $d$ levels is large, $\mu_{0}-\epsilon_{0} \gg\left|Q_{12}\right|$, or the temperature is high enough $k T \gg\left|Q_{12}\right|\left(Q_{12}\right.$ is defined below). The temperature dependent exchange integral is equal to

$$
J(\boldsymbol{R}, \mu, T)=\frac{1}{2 \beta S^{2}} \sum_{\omega_{m}} \frac{Q_{12}\left(\omega_{m}\right) Q_{21}\left(\omega_{m}\right)}{\left(-\mathrm{i} \hbar \omega_{m}+\mu_{0}-\epsilon_{0}+Q_{11}\left(\omega_{m}\right)\right)^{2}}
$$

where $\omega_{m}=(2 m+1) \pi / \beta$ are Matsubara frequencies and

$$
Q_{i \sigma, j \sigma^{\prime}}\left(\omega_{m}\right)=\sum_{k, \alpha} V_{k \alpha, j \sigma^{\prime}} V_{k_{\alpha, i \sigma}}^{*} /\left(\mathrm{i} \omega_{m}-\epsilon_{\boldsymbol{k}}+\mu\right) \text {. }
$$

With the assumptions concerning the band structure and hybridization presented in Sec. 2 we obtain the following formulas for $Q_{12}\left(\omega_{m}\right)=Q_{21}\left(\omega_{m}\right)$ and $\operatorname{Im} Q_{11}\left(\omega_{m}\right)$ (we neglect the real part of $Q_{11}\left(\omega_{m}\right)$ because it leads only to the shift of $\left.\epsilon_{0}\right)$. The imaginary part of $Q_{11}$,

$$
\operatorname{Im} Q_{11}(\omega)=-\operatorname{sgn}(\omega)\left(3 V^{2} a_{0}^{3}\right) /(2 \pi)\left(2 m / \hbar^{2}\right)^{3 / 2}\left(\sqrt{\mu_{0}-i \hbar \omega}+\text { c.c. }\right),
$$

may be interpreted as the width of the $d$ level caused by the hybridization with the valence band. If we define

$$
Q(r)=\left(m V^{2} a_{0}^{3}\right) /\left(2 \pi r \hbar^{2} \sqrt{A}\right) \exp \left[-\mathrm{i} r \sqrt{2 m\left(\mu_{0}-\mathrm{i} \hbar \omega\right) / \hbar^{2}}\right],
$$

then

$$
Q_{12}\left(\omega_{m}\right)=4\left[\cos \left(K_{0} R_{x}\right) \cos \left(K_{0} R_{y}\right) Q\left(r_{z}\right)+\text { cycl. perm. of }(x, y, z)\right],
$$

where $r_{x}=\sqrt{R_{x}^{2} / A+R_{y}^{2}+R_{z}^{2}}$ and similarly for $r_{y}$ and $r_{z}$. 


\section{Results}

The simplest way to calculate $J(\boldsymbol{R}, \mu, T)$ is the direct numerical computation of the sum in Eq. (1). For $T \rightarrow 0$ it is possible to present the closed formula for $J(\boldsymbol{R}, \mu, T)$ in terms of exponential integrals of complex arguments. However, that formula is very complicated and nontransparent and that is why is not presented here. The general features of $J(R, \mu, T)$ are following. It is an oscillating function of $R$ which for $R \rightarrow 0$ behaves like $1 / R$ and for $r \rightarrow \infty$ like $1 / R^{3}$. The amplitude of the oscillations is proportional to the squared mass of the carriers, fourth power of $V$ and decreases with $\mu_{0}-\epsilon_{0}$ which is the main result of the paper. The behavior of $J(R, \mu, T)$ is practically temperature independent for $\mu-\epsilon_{0}>k T+\operatorname{Im} Q_{11}$, i.e. when the $d$ level is occupied only virtually. If $\mu-\epsilon_{0}$ becomes comparable with the width of the $d$ level or with the temperature broadening of the Fermi level the behavior changes. This is particularly well seen in Fig. 1 where the inverse susceptibility versus temperature is presented (in calculation of the Curie temperature forty coordination zones were taken into account).

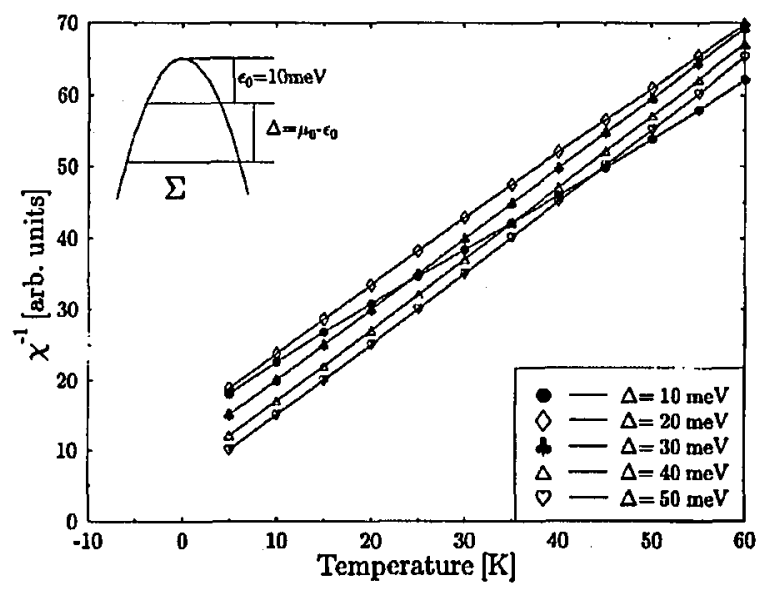

Fig. 1. The calculated inverse susceptibility versus temperature for different distances between the Fermi and $d$ levels for $V=100 \mathrm{meV}$.

The model presented above has only qualitative meaning. It is too simple to describe experimental results quantitatively. Firstly, the band parameters $m, A$, $K_{0}$ are known only approximately. Secondly, as was already pointed out the form of $V_{k \alpha, i \beta}$ does not take into account many effects, in particular the extension of the $d$ orbital or the spin-orbit interaction.

Despite all the deficiencies the model predicts properly the variation of the paramagnetic temperature with the free carrier concentration. The other prediction, i.e. the variation of the exchange integral with the temperature, at present, is not confirmed experimentally. However, the existing experimental data does not exclude it. 


\section{References}

[1] M. Górska, T. Story, M. Arciszewska, E. Grodzicka, Z. Gołacki, A. Eusakowski, Acta Phys. Pol. A 87, 197 (1995).

[2] T. Story, M. Górska, M. Arciszewska, E. Grodzicka, Z. Gołacki, R.R. Gałazka, J. Magn. Magn. Mater. 140-144, 2041 (1995).

[3] T. Kasuya, IBM J. Res. Dev. 14, 214 (1970).

[4] T. Story, P.J.T. Eggenkamp, C.H.W. Swuste, H.J.M. Swagten, W.J.M. de Jonge, L.F. Lemmens, Phys. Rev. B 45, 1660 (1992) and references therein.

[5] J.W. Negele, H. Orland, Quantum Many-Particle Systems, Addison-Wesley Publ. Comp., Redwood City 1988. 